\title{
Analysis of unsteady behaviour in shockwave turbulent boundary layer interaction
}

\author{
Muzio Grilli ${ }^{1} \dagger$, Peter J. Schmid ${ }^{2}$, Stefan Hickel ${ }^{1}$ and Nikolaus A. Adams ${ }^{1}$ \\ ${ }^{1}$ Institute of Aerodynamics and Fluid Mechanics, Technische Universität München, \\ D-85748 Garching, Germany \\ ${ }^{2}$ Laboratoire d'Hydrodynamique (LadHyX), CNRS-École Polytechnique, 91128 Palaiseau, France
}

(Received 16 June 2011; revised 13 December 2011; accepted 17 January 2012; first published online 28 February 2012)

\begin{abstract}
The unsteady behaviour in shockwave turbulent boundary layer interaction is investigated by analysing results from a large eddy simulation of a supersonic turbulent boundary layer over a compression-expansion ramp. The interaction leads to a very-low-frequency motion near the foot of the shock, with a characteristic frequency that is three orders of magnitude lower than the typical frequency of the incoming boundary layer. Wall pressure data are first analysed by means of Fourier analysis, highlighting the low-frequency phenomenon in the interaction region. Furthermore, the flow dynamics are analysed by a dynamic mode decomposition which shows the presence of a low-frequency mode associated with the pulsation of the separation bubble and accompanied by a forward-backward motion of the shock.
\end{abstract}

Key words: compressible boundary layers, shock waves

\section{Introduction}

The shock-wave turbulent boundary layer interaction (SWTBLI) is one of the most prevalent phenomena occurring in high-speed flight. From an engineering point of view, this phenomenon can have a significant impact on aircraft or rocket performance. The interaction of the shock with the turbulent boundary layer causes the separation of the boundary layer and the subsequent formation of a recirculation region. The main characteristic of such an interaction is the low-frequency unsteadiness of the shock producing amplified wall pressure fluctuations which, in turn, can cause strong buffeting of the underlying structure, eventually leading to failure due to fatigue.

Several experimental investigations on impinging shock or compression ramp configurations have been carried out over the last few decades, focusing their attention on the fluctuating nature of the wall-pressure signal in the interaction region (Dolling \& Murphy 1983; Dolling \& Or 1985; Beresh, Clemens \& Dolling 2002; Ganapathisubramani, Clemens \& Dolling 2006, 2009; Dupont, Haddad \& Debieve 2006). Considerable contributions to our understanding of such a phenomenon also came from numerical studies. Direct numerical simulations (DNSs) (Adams 2000; Pirozzoli \& Grasso 2006; Wu \& Martin 2007) and large eddy simulations (LESs) (Loginov, Adams \& Zheltovodov 2006; Touber \& Sandham 2009) of the 
shock/boundary-layer interaction started to appear in the last decade. Such studies have unanimously confirmed the low-frequency motion of the shock whose characteristic time scale is two or three orders of magnitude smaller than the characteristic time scale of the incoming boundary layer (Dolling \& Murphy 1983), which is $O\left(\delta / U_{\infty}\right)$.

Despite a large number of experimental and numerical studies aimed at characterizing the shock oscillations and identifying large-scale turbulence, the exact cause of the low-frequency motion still remains an open question. At present, there is no general agreement on the interpretation of numerical or experimental findings. Two main lines of thought seem to emerge. On one side, based on time-resolved particle-image velocimetry (PIV) and Taylor's hypothesis, Ganapathisubramani et al. (2006) reported the presence of very long coherent structures with a length of about 50 boundary-layer thicknesses in the incoming, turbulent boundary layer. According to their interpretation, the passage of such 'superstructures' is directly responsible for the motion of the shock. A simple scaling argument for the low frequency induced by such structures, i.e. $U_{\infty} /\left(2 L_{s}\right)$ with $U_{\infty}$ as the upstream free stream velocity and $L_{s}$ as the size of the structure, was able to recover the typical frequency range of the shock motion. Nevertheless, the origin of such superstructures remains unclear. Support for this hypothesis is provided by the DNS results of Ringuette, Martin \& Smits (2006) where long coherent structures with a length of $48 \delta_{0}$ have been observed. However, the appearance of such structures could be related to the recycling/rescaling technique used in this work to generate the incoming turbulent boundary layer. A second line of thought states that the low-frequency oscillation is due to a coupling mechanism between the separation bubble and the shock (Dupont et al. 2006; Dussauge, Dupont \& Debieve 2006; Piponniau et al. 2009; Touber \& Sandham 2009). Dupont et al. (2006) measured the characteristic frequency of the shock motion for a $M a=2.3$ oblique shockwave for several angles of flow deflection $\left(7^{\circ}<\theta<9.5^{\circ}\right)$, and found that for an identical upstream turbulent boundary layer the shock frequency is directly related to the intensity of the interaction. They obtained a typical Strouhal number, based on the length of interaction $L_{i}$ and the velocity outside of the separation bubble $U_{\infty}$, as $S_{L_{i}}=f L_{i} / U_{\infty} \approx 0.03$. In extending the work of Dupont et al. (2006), Piponniau et al. (2009) introduced a model to explain the low-frequency unsteadiness found in shock-induced separation for cases where the flow reattaches downstream. They based their model on the properties of fluid entrainment in the mixing layer generated downstream of the reflected shock. Furthermore, using conditional analysis of their PIV data, they showed that the contractions of the bubble are related to downstream motion of the reflected shock, whereas the expansions are related to intense reverse flow and, consequently, upstream motion of the reflected shock. Support for such a mechanism came also from numerical investigations. Touber \& Sandham (2009) performed a linear-stability analysis of the base flow extracted from a LES that matches the experiment. The analysis showed the presence of a two-dimensional, nonoscillatory but globally unstable mode whose temporal growth rate was found to be substantially smaller than the typical inverse time scales of the incoming turbulent boundary layer. An important contribution to the understanding of the SWTBLI phenomenon came from Pirozzoli et al. (2010), who analysed their impinging shock LES database by using Koopman modes and linear global stability analysis. They identified two distinct modes, one linked to the turbulent structures in the incoming boundary layer, the other associated with a cyclic expansion and contraction of the separation region.

The main objective of the present work is to investigate the unsteady features of the SWTBLI in order to extract the main properties of the mechanism that yields low- 
frequency shock unsteadiness. For this purpose, results from a large-eddy simulation of a supersonic turbulent boundary layer over a compression-expansion ramp are analysed by means of the dynamic mode decomposition (DMD) (Schmid 2010). Details of the numerical test case are described in $\S 2.1$, and the main features of the flow are presented in $\S 2.3$. Characteristics of the shock motion are first analysed in terms of wall-pressure data in $\S 3.1$, after which a modal analysis is presented in $\S 3.2$. Conclusions are provided in $\S 4$.

\section{Numerical database}

\subsection{Flow configuration}

The flow conditions are matched to an experiment of Zheltovodov et al. (1990), characterized by a free stream Mach number of $M a_{\infty}=2.88$ and a Reynolds number based on the incoming boundary layer thickness of $R e_{\delta_{0}}=132840$. The computational domain, sketched in figure $1(a)$, has dimensions $L_{x}=47.845 \delta_{0}, L_{y}=12 \delta_{0}$ and $L_{z}=$ $4 \delta_{0}$. The computational grid has been generated using an adaptive-mesh-refinement procedure which ensures that the first grid point in the wall-normal direction at the inlet is located at $y^{+} \approx 2.2$. The total number of points in wall normal direction in the inflow plane is 300 . Six successive steps with a refinement factor of two are employed. Sixty points are employed in the region $0<y / \delta_{0}<0.25$, 60 points in the region between $0.25<y / \delta_{0}<0.75,45$ points in the region $0.75<y / \delta_{0}<1.5$, 45 points in the region $1.5<y / \delta_{0}<3.0,45$ points in the region $3.0<y / \delta_{0}<6.0$ and 45 points in the region $3.0<y / \delta_{0}<12.0$. Such a resolution has been found adequate to reproduce the experimental results in the first reference section $E 1$ accurately. The grid spacing in the streamwise direction is $\Delta x^{+} \approx 52.9$, and in the spanwise direction a resolution of $\Delta z^{+} \approx 25.7$ has been adopted. A total number of $32.5 \times 10^{6}$ grid points are used to discretize the computational domain. The reference length throughout this work is the mean boundary-layer thickness of the experiment $\left(\delta_{0}=4.1 \mathrm{~mm}\right)$ at the first reference section $E 1$. In what follows, dimensional quantities are indicated by an asterisk; the non-dimensionalization then reads

$$
\left.\begin{array}{l}
u_{i}=u_{i}^{*} / U_{\infty}^{*}, \quad \rho=\rho^{*} / \rho_{\infty}^{*}, \quad T=T^{*} / T_{\infty}^{*}, \\
p=p^{*} /\left(\rho_{\infty}^{*} U_{\infty}^{* 2}\right), \quad E=E^{*} /\left(\rho_{\infty}^{*} U_{\infty}^{* 2}\right) .
\end{array}\right\}
$$

Reference data are taken from the experiment as $U_{\infty}^{*}=618.1 \mathrm{~m} \mathrm{~s}^{-1}, \rho_{\infty}^{*}=$ $0.368 \mathrm{~kg} \mathrm{~m}^{-3}$ and $T_{\infty}^{*}=114.8 \mathrm{~K}$. Thirteen different experimental measurement sections are considered for the validation of our numerical results. A sketch indicating the locations of the measurement sections along the compression/expansion ramp is given in figure $1(b)$. A comparison with the experimental data of the integral features of the incoming turbulent boundary layer is given in table 1. Figure 2(a) shows mean profiles of velocity, temperature, density and Mach number for the experiment and the present computation. Corresponding Reynolds stress profiles are presented in figure 2(b), where also the DNS data of Spalart (1988) is shown for comparison.

\subsection{Numerical approach}

The three-dimensional, compressible Navier-Stokes equations in conservative and non-dimensionalized form are solved numerically with the assumption of an ideal gas and a power-law dependence of the viscosity and thermal conductivity on the temperature. The governing equations are discretized using the compressible adaptive local deconvolution method (ALDM) for the convective fluxes (Hickel 2012), see 
(a)

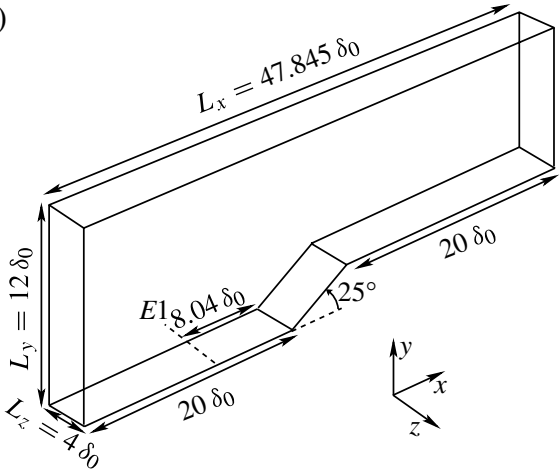

(b)

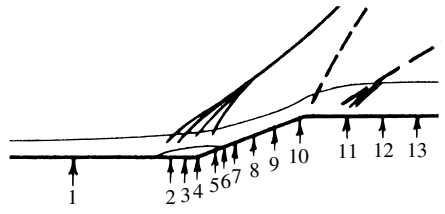

FIGURE 1. (a) Sketch of the computational domain and dimensions for the LES. $(b)$ Locations of the experimental measurement probes along the lower wall.

$\begin{array}{lcccccc} & \delta_{0} & \delta_{1} & \delta_{2} & \operatorname{Re}_{\delta_{0}} & \operatorname{Re}_{\delta_{2}} & C_{f} \times 10^{3} \\ \text { Experiment } & 1.0 & 0.349 & 0.073 & 132840 & 4924 & 1.47 \\ \text { Computation } & 1.0 & 0.314 & 0.079 & 132846 & 5385 & 1.41\end{array}$

TABLE 1. Summary of mean-flow parameters for the flat-plate boundary layer simulation.
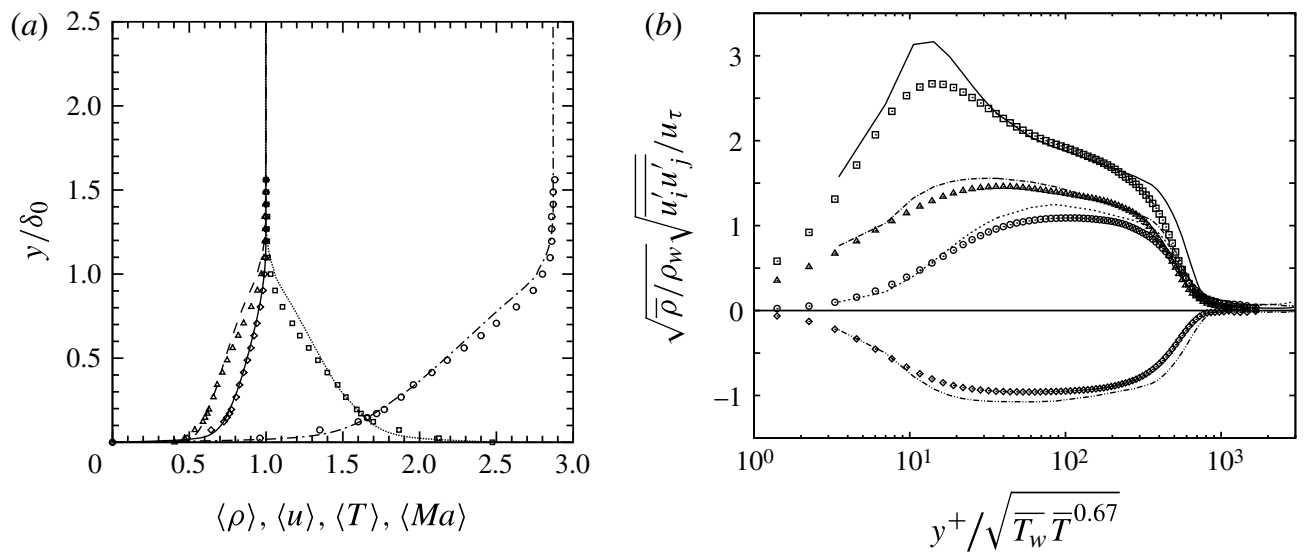

FIGURE 2. (a) Wall normal distribution of the mean flow for the incoming boundary layer at the station $E 1$. Mach number $(-\bullet-$, LES; o, experiment); temperature $(\cdots \cdots$, LES; $\square$, experiment); velocity (—, LES; $\diamond$, experiment); density (- - , LES; $\triangle$, experiment). (b) Turbulence statistics at the station E1. Lines denote LES data shown in inner-layer scaling using the compressibility correction of Huang, Coleman \& Bradshaw (1995) and symbols represent DNS data of Spalart (1988).

also (Hickel, Adams \& Domaradzki 2006), which provides a physically consistent implicit subgrid-scale (SGS) model. ALDM is based on a nonlinear finite-volume scheme with a solution-adaptive deconvolution operator and a numerical flux function. 

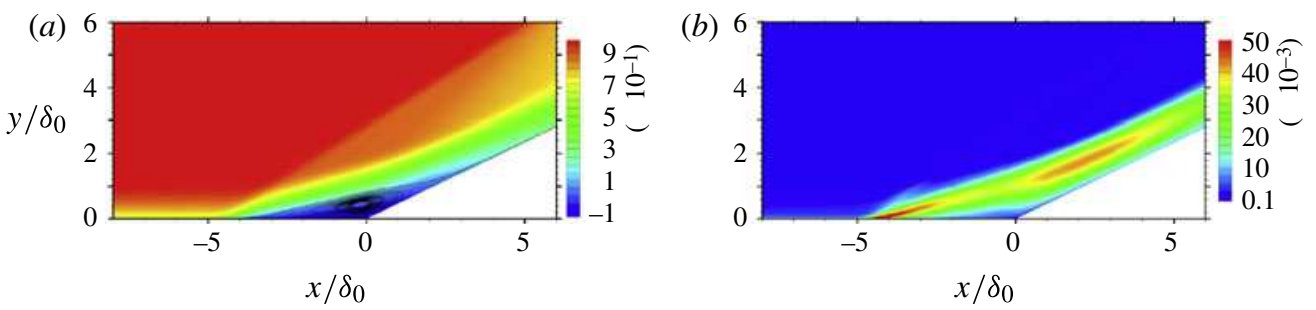

FIgURE 3. (a) Contours of the mean streamwise velocity field in the region of interaction, with 23 contour levels ranging from -0.1 to 1.0. Streamlines are indicating the recirculating flow region. (b) Streamwise-velocity fluctuation field $\left\langle u^{\prime} u^{\prime}\right\rangle / U_{\infty}^{2}$ in the region of interaction, with 23 contour levels ranging from 0 to 0.058 .

Model parameters allow control of the truncation error and have been calibrated such that the truncation error acts as a physically consistent SGS model. A second-order central difference scheme is used to compute the diffusive fluxes and a third-order Runge-Kutta scheme to integrate in time. Periodic boundary conditions are imposed in the spanwise direction, whereas an immersed interface method is applied to describe the compression ramp geometry, which reproduces an adiabatic no-slip wall. The top (free stream) and outflow boundaries make use of a linear extrapolation procedure. A synthetic-turbulence inflow technique (Touber \& Sandham 2009) is implemented, which is designed to match given first- and second-order statistical moments and spectra. The adoption of such a technique avoids the introduction of low frequencies into the computational domain due to artificially correlated data, therefore eliminating spurious interferences with the SWTBLI.

\subsection{Statistical flow properties}

Figure 3(a) shows a close-up view of the mean streamwise-velocity field in the interaction region. The incoming undisturbed boundary layer is affected by the separation shock, and the interaction results in the appearance of a reverse flow region and of a separated shear layer above. Figure 3(b) displays a close-up view of the streamwise-velocity fluctuations in the area of interest. In accordance with previous numerical studies (Adams 2000; Loginov et al. 2006; Wu \& Martin 2007), the interaction of the shock with the boundary layer produces an amplification of the Reynolds stresses past the interaction zone, which can be associated with the generation of a mixing layer above the separation region. In order to further validate the numerical database adopted for our analysis, a comparison between numerical and experimental results in terms of the wall pressure $p_{w}$ and the skin friction coefficient $C_{f}$ is given in figure 4 . The averaged wall pressure, normalized by its mean value at the station $E 1$, monotonically increases over the extent of the shockwave/boundary layer interaction region, with a plateau inside the separation zone. Further downstream, it decays to the initial values in the expansion fan, see figure $4(a)$. The slight pressure rise in the region $8<x / \delta_{0}<9$ may indicate the existence of a weak compression wave inside the boundary layer downstream of the expansion. The mean skin friction exhibits the typical behaviour for separated flow, figure $4(b)$. After the initial transient due to the inflow turbulence generation technique, the typical decrease of $C_{f}$ along the flat plate is visible which is consistent with boundary-layer growth and increase of local Reynolds number, $x / \delta_{0}<-6$. The friction coefficient drops abruptly near the separation point $(\mathbf{S})$ to negative values inside the reverse-flow region 

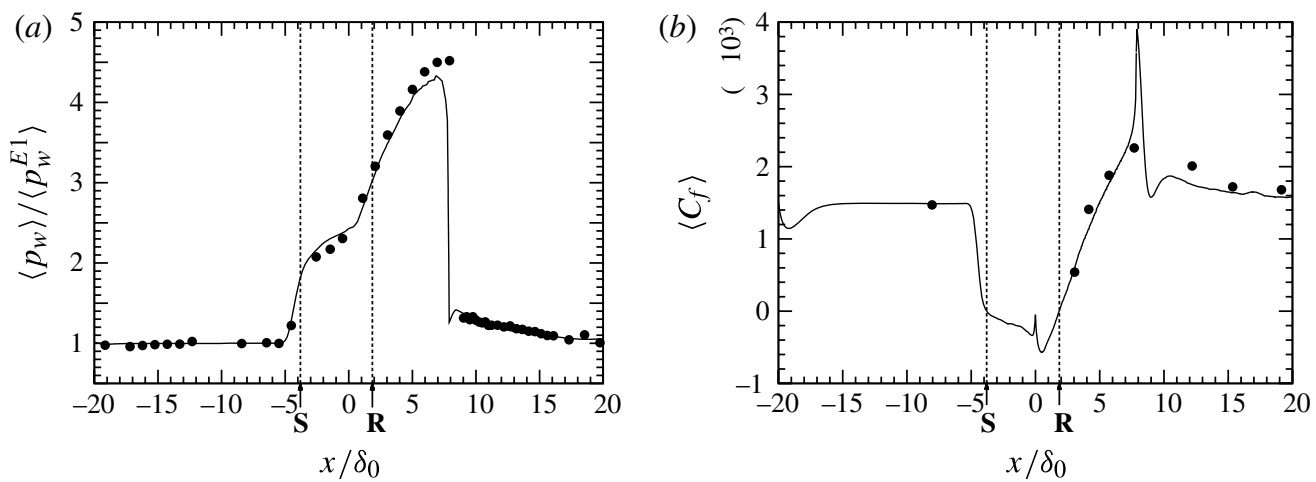

FIGURE 4. Averaged wall-pressure $(a)$ and skin-friction coefficient $(b)$ distributions versus the streamwise direction. The symbols indicate the reference experiment, the solid line represent results from the LES, averaged in time and over the spanwise direction. The labels $\mathbf{S}$ and $\mathbf{R}$ mark the mean separation and reattachment point, respectively.
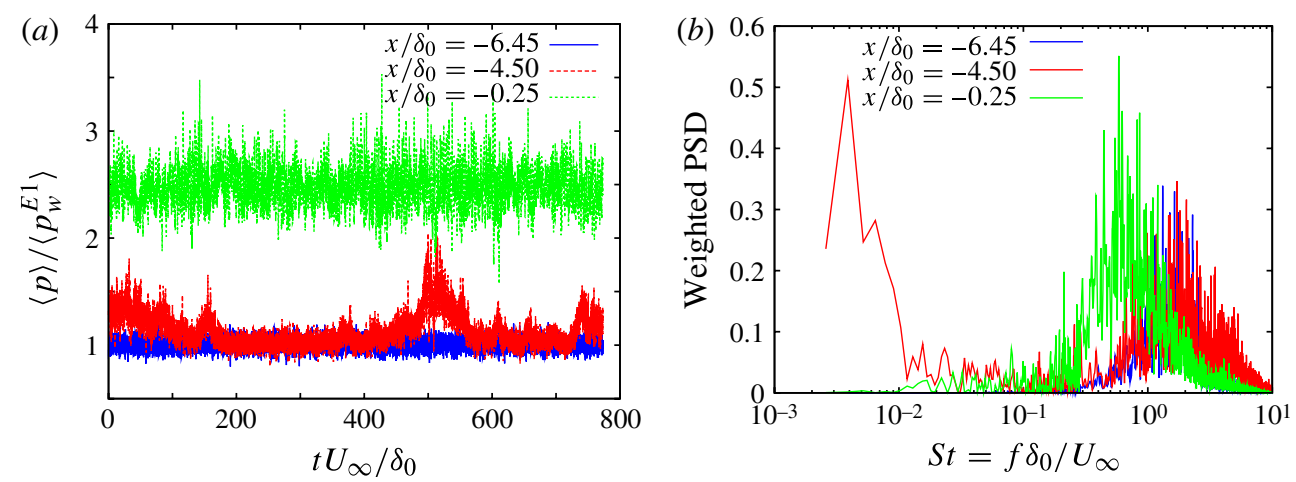

FIGURE 5. (a) Wall-pressure signals and (b) corresponding weighted power spectral density at different streamwise locations relative to the ramp corner.

$-4.5<x / \delta_{0}<2.0$. It rises again after the reattachment point $(\mathbf{R})$, reaching values on the upper surface, which are slightly larger than that of the incoming boundary layer.

\section{Shock motion}

\subsection{Analysis of the wall-pressure signal}

We start by analysing LES wall-pressure signals from 130 pressure probes placed along the median line of the wall. The probes are equally distributed within a region that extents from $x / \delta_{0}=-6.5$ (upstream of the shock foot position) to $x / \delta_{0}=0$ (the ramp corner) with a spacing of $\Delta x / \delta_{0}=0.05$. The pressure signals have been recorded for each time step.

Figure 5(a) shows wall-pressure signals at three different positions, highlighting the characteristics of the shock motion. In the incoming boundary layer at $x / \delta_{0}=-6.45$, the normalized magnitude is around unity with only small fluctuations. At $x / \delta_{0}=-4.5$ the signal fluctuates between 1 and 2, showing peaks which are due to the motion of the shock. At position $x / \delta_{0}=-0.25$, which is located in the separation region, the mean-pressure value increased to 2.5 , but distinct peaks are no longer 


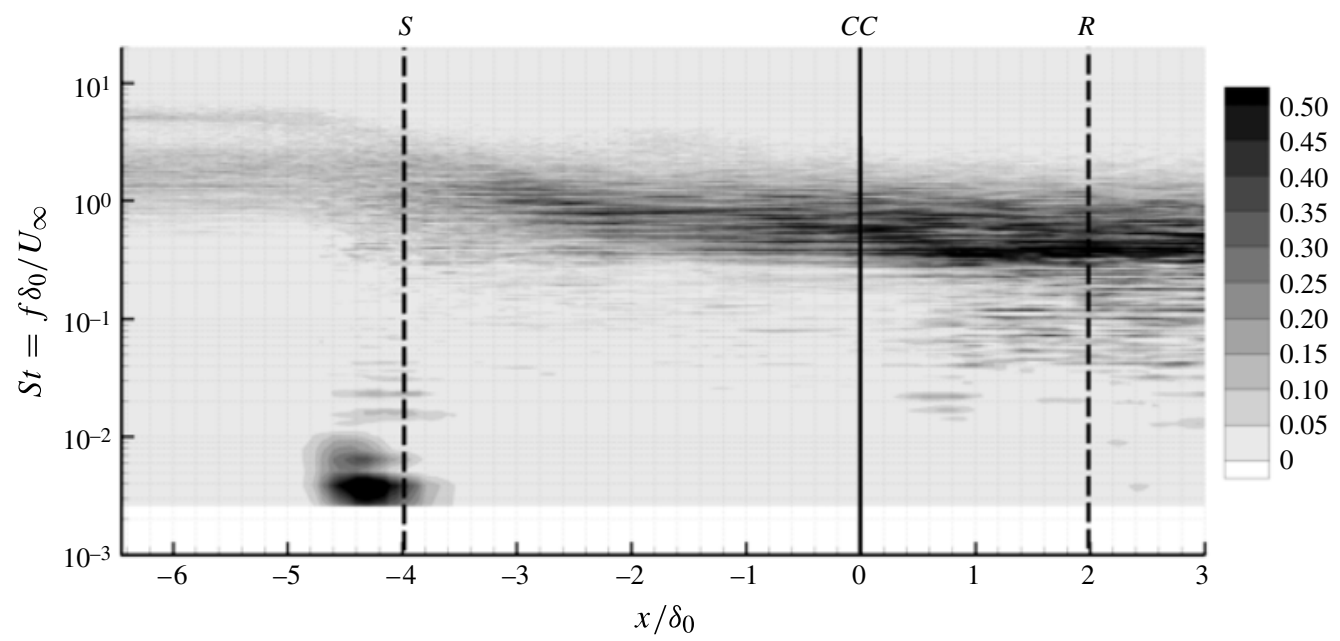

FIGURE 6. Contours of the weighted power spectral density of wall pressure in the $S t-x / \delta_{0^{-}}$ plane. The power spectral density is normalized such that their integral over frequency is unity. Thirty contours levels have been used; the dashed vertical lines indicate the location of the separation $S$ and reattachment point $R$ respectively. The solid line indicates the position of the corner $C C$.

visible. Nonetheless, we have an increase of the pressure-fluctuation amplitude which corresponds to turbulence amplification by the shock. The corresponding weighted power spectral densities are plotted in figure $5(b)$. At the shock foot the peak frequency is $0.0039 U_{\infty} / \delta_{0}$. Given the amount of available data from the simulation, a total number of three cycles of shock motion are considered in the present analysis. A Strouhal number $S t_{L}=f L / U_{\infty}$ can be defined, where $L$ is the length of the mean separation bubble $\left(L=6.0 \delta_{0}\right.$ in the present LES), and gives $S t_{L}=0.0234$ which is consistent with the range of $S t_{L}$ indicated by Dussauge et al. (2006).

The unsteady character of the interaction zone is further illustrated by the analysis of the wall-pressure spectra, shown in figure 6 as a function of the Strouhal number and the streamwise coordinate direction. Upstream of the interaction, the spectra show typical features of turbulent boundary layers with the dominant peak located at $S t \approx 1$. A qualitative change is observed near the foot of the shock (highlighted with a dashed line in the figure) where substantial energy at lower frequencies is found, which confirms the low-frequency nature of the unsteadiness in this region. The low-frequency peak disappears when moving towards the centre of the separation region and, approaching the reattachment location, the energy is evenly distributed over three decades of Strouhal numbers. This behaviour is in good agreement with results reported by Dupont et al. (2006).

\subsection{Dynamic mode decomposition}

Dynamic mode decomposition is a technique that allows for a modal analysis of a data sequence, without resorting to a numerical solver or an underlying model (Schmid 2010, 2011). In the case of a linearized flow (i.e. a flow of small perturbation about a steady base flow), the extracted structures are equivalent to global eigenmodes. For a nonlinear flow, the decomposition produces modes that express the dominant dynamic behaviour captured in the data sequence. Defining a temporal linear operator $A$ which 

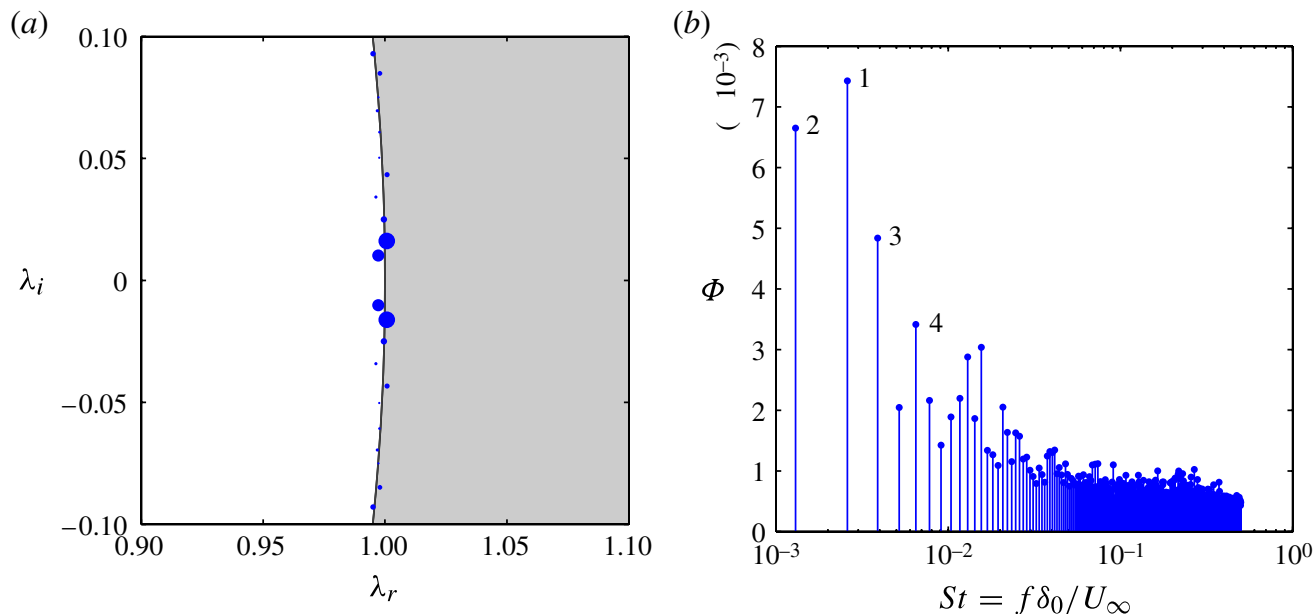

FIgURE 7. (a) Close-up view of the eigenvalue distribution of the inter-snapshot mapping in the complex plane, detected by the DMD method. (b) Amplitude distribution of the detected modes.

advances our snapshot basis $\mathrm{V}(t)$ such that $\mathrm{V}\left(t^{n+1}\right)=\mathrm{AV}\left(t^{n}\right)$, the DMD algorithm is able to extract from the data sequence approximate eigenvalues and eigenmodes of a reduced-order representation of the linear operator A. For sufficiently long sequences, each DMD-mode is then linked to a single temporal frequency (Rowley et al. 2009). In this manner, DMD differs from the proper orthogonal decomposition which generally does not yield direct information concerning the frequency of the detected modes. This feature makes DMD analysis particularly suited to the present study, since our goal is to focus on low-frequency unsteadiness. The DMD algorithm has thus been applied to temporal snapshots of the spanwise-averaged flow variables in a subdomain containing the interaction region and the recirculation bubble. The box used for the DMD extraction has the exact dimensions of the contour plot shown in figure 8 . The box extends in the range $-4.5<x / \delta_{0}<2.4$ in the streamwise direction and in the range $0<y / \delta_{0}<2$ in the wall normal direction. A total number of 770 snapshots, equispaced in time with $\Delta t U_{\infty} / \delta_{0}=1$ (allowing for a resolvable Strouhal number of 0.5 ), have been processed. The choice of sampling interval has been motivated by our aim of extracting the low-frequency unsteadiness; the highfrequency part of the spectrum is under-sampled. Moreover, it has been verified that reducing the frequency of the sampling for the DMD extraction does not affect the low-frequency high-amplitude modes, allowing us to state that these modes are not biased by aliasing effects. It has also been verified that the superposition of all of the obtained DMD modes recovers a flow field approximately resembling that of the full LES. The eigenvalues $\lambda$ of the reduced-order representation of the linear inter-snapshot mapping A are shown in figure $7(a)$. All eigenvalues fall on the unit circle, which is expected for a saturated system. Figure 7(b) shows the amplitude distribution for the detected modes, plotted versus the dimensionless frequency. The Strouhal number of the maximum-amplitude mode is found to be 0.00282, in good agreement with the value obtained from the Fourier analysis of the wall-pressure data.

A reconstruction of the flow field based on the four most dominant modes (labelled in figure $7(b)$ ) has been carried out where the flow field evolution of each mode has been recovered based on its frequency and amplitude and then added to the mean flow; 


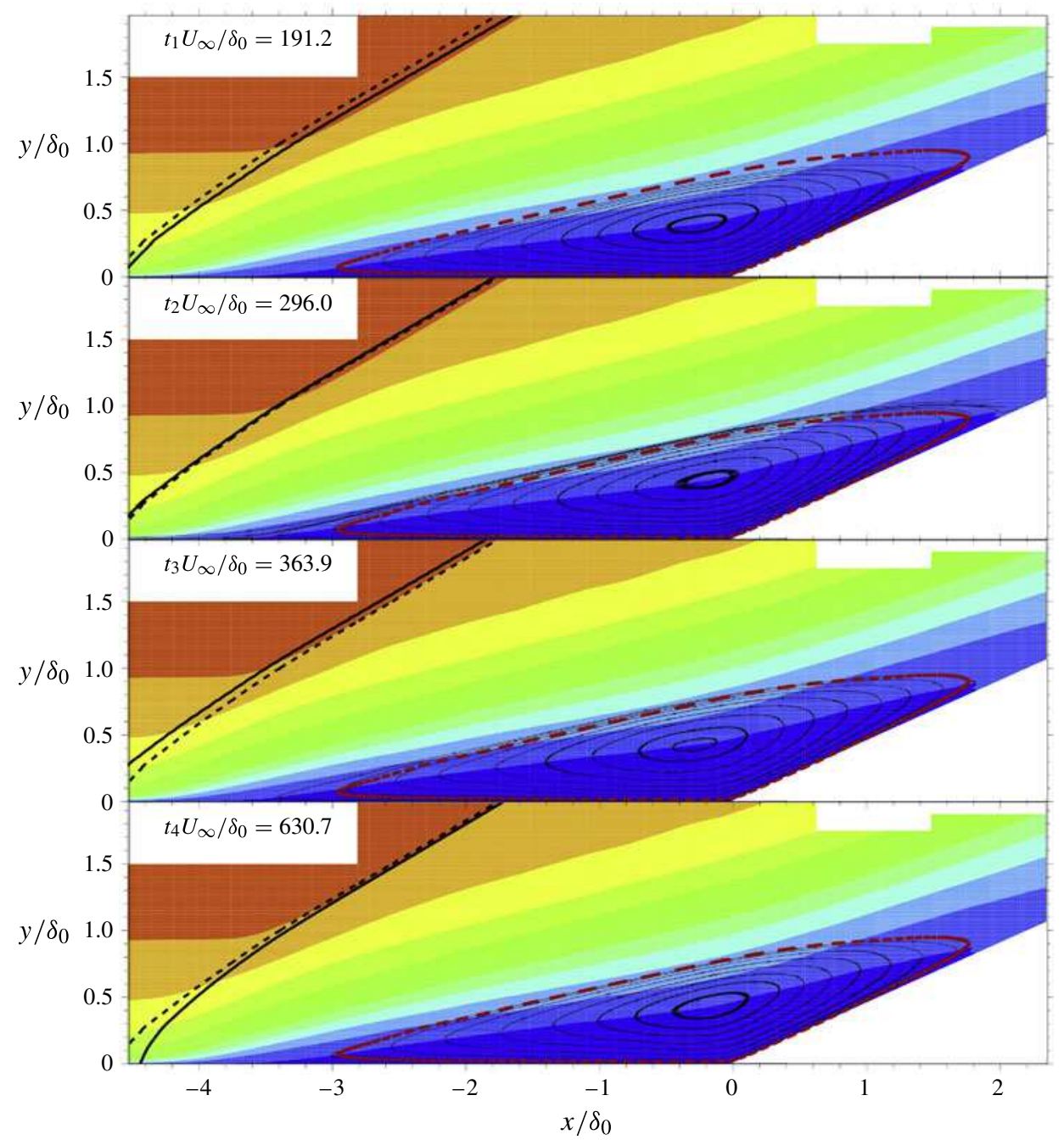

FIGURE 8. Four snapshots from a reconstruction of the flow fields from a linear combination of the four most dominant dynamic modes. - Instantaneous oblique shock position; - Mean oblique shock position. - - , Mean recirculation region domain.

figure 8 shows the result, displayed at four time instants. The mean position of the shock and the mean recirculation region are indicated by dashed lines. The choice of the number of modes to be considered was based on the decrease of the error norm obtained by adding another mode to the reconstruction. The norm was computed as $\sum_{t=0}^{t=2 T}\left|s^{i+1}(t)-s^{i}(t)\right| /\left|s^{i}(t)-s^{1}(t)\right|$, where $i$ equals the number of modes considered, and $T$ is the period of the mode having the highest amplitude. With four DMD modes the error norm for the reconstructed fields is below 15\%. The reconstructed flow exhibits a breathing motion, with the separation bubble expanding and shrinking periodically and the shock moving forward-backward accordingly. Owing to the low-frequency nature of the selected modes no structures reminiscent of turbulence can be detected in the reconstruction. 
In order to further investigate the features of the low-frequency unsteadiness, the shock location and the mass of the recirculation region versus time have been determined for the reconstructed flow. The four most dominant modes have successively been added one after the other to the mean flow, and mass of the recirculation region as well as the shock displacement have been computed for each resulting flow field. The shock position was tracked away from the wall at $x / \delta_{0}=-1.95$ and $y / \delta_{0}=1.75$. This location allows for a more precise definition of the shock position as there it is sharply developed and less affected by boundarylayer turbulence. The exact position of the shock was determined by computing the location of the maximum density gradient in $x$-direction. It should be noted that the extent of the shock motion reduces while moving away from the wall (Dupont et al. 2006), thus that the extent of the shock motion as reported in the figure $9(b)$ is lower than typical shock-excursion values reported for the shock foot at the wall. An estimate of the actual value of the shock-motion amplitude in the proximity of the wall can be extracted from figure 6 , where the region indicating high levels of energy in the low-frequency regime extents from $x / \delta_{0}=-4.75$ to $x / \delta_{0}=-3.5$, giving a value for the shock-motion amplitude of $1.25 x / \delta_{0}$. The mass of the reverse flow region has been computed by considering the region of the flow where the $u$-velocity is negative. As can be seen in figure $9(a)$ the superposition of the modes confirms a temporal asymmetry in the expansion-contraction cycle of the bubble and the oscillatory behaviour of the shock. With all four modes superposed on the mean flow, we observe that the mass increase in the recirculation region occurs significantly faster than the subsequent mass loss. Simultaneously, the downstream displacement of the shock is slower than its upstream motion. This is consistent with the model proposed by Piponniau et al. (2009) in which contractions of the bubble stem from a mass entrainment process involving the shear layer above the recirculation region, whereas the dilatations correspond to a large mass flux in the reverse direction, needed to maintain the recirculation region. In addition, a clear delay between changes of the recirculation region and the motion of the shock can be detected; the instants in time of respective minima and maxima are indicated in figure $9(a)$. An animation of the reconstructed flow field by the four dominant modes, available as a supplement to the online version at journals.cambridge.org/flm, clearly highlights the modification of the separation bubble as the main driver of the shock motion.

\section{Summary and conclusions}

We have applied a dynamic mode decomposition to a set of snapshots of spanwiseaveraged flow fields obtained by a LES of a supersonic turbulent boundary along a compression-expansion ramp. By considering a subdomain that contains both the shock and the recirculation region, we have identified and isolated a high-amplitude mode within the low-frequency regime. A temporal reconstruction of the flow field associated with the four most dominant modes reproduces a pulsating separation bubble together with an oscillatory streamwise motion of the shock. This reduced description of the flow behaviour (based on four dynamic modes) captures the main features of the shock-wave turbulent-boundary-layer interaction: a clear coupling between the motion of the recirculation region and the motion of the shock in agreement with experimental observations (Piponniau et al. 2009). Our results show that SWTBLI cannot be described by a single mode, but that the superposition of the four dominant low-frequency modes is sufficient to recover the essential characteristics. These modes are phase locked and represent an asymmetric cycle for the shock motion 

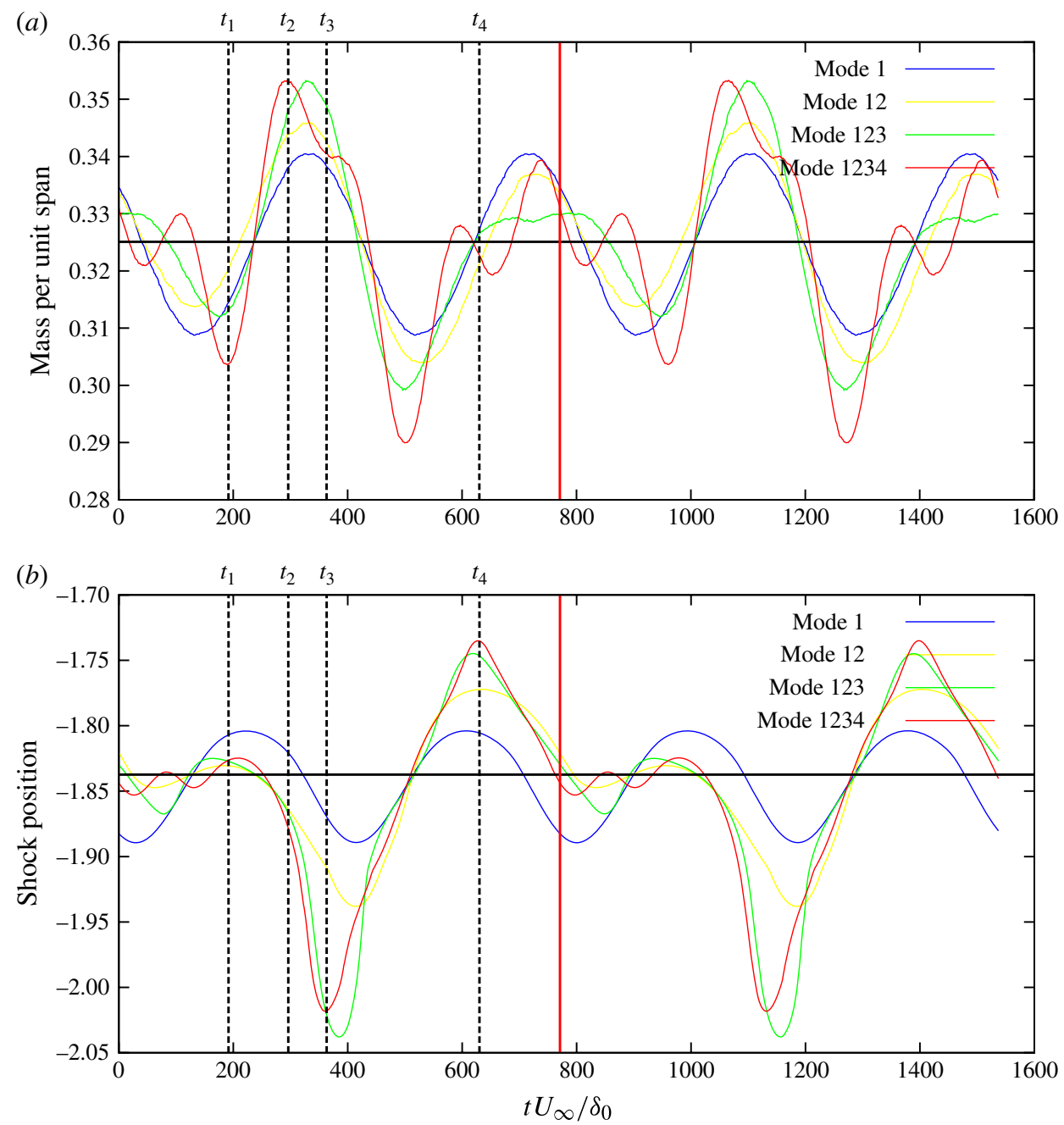

FiguRE 9. (a) Temporal evolution of the mass (per unit span) of the reverse-flow region; $(b)$ shock location versus time. Each variable has been extracted from a reconstructed flow field sequence based on the four most dominant dynamic modes.

as well as the periodic pumping of the separation bubble. An upstream extension of the domain considered for the DMD analysis showed negligible variations of the flow field in the region located before the interaction. Based on this reconstruction we can state that upstream effects of the incoming boundary layer are irrelevant for the SWTBLI considered in our investigation since adopting the scaling argument introduced by Ganapathisubramani et al. (2006) the length of the structure able to generate such a low frequency would exceed the domain considered upstream of the interaction in our simulation. At the same time, any of the structures that could be generated upstream would infer a low-frequency motion which is at least one order of magnitude higher than any of the four DMD modes considered in our analysis. Based on this evidence we conclude that such structures cannot represent the driving mechanism for the low-frequency motion of the shock. Our results fully support the hypothesis that the observed SWTBLI phenomena are a consequence of 
the inherent dynamics between separation bubble and shock and are not driven by upstream coherent structures.

\section{Acknowledgements}

Financial support has been provided by the German Research Foundation (DFG) through the SFBTRR 40 project. Computational resources have been provided by the Leibniz-Rechenzentrum München (LRZ). The authors acknowledge the support of the TUM Graduate Schools Faculty Graduate Center Mechanical Engineering. The authors would also like to thank Professor R. Friedrich for fruitful discussions.

Supplementary movies are available at journals.cambridge.org/flm.

\section{REFERENCES}

ADAms, N. A. 2000 Direct simulation of the turbulent boundary layer along a compression ramp at $M a=3$ and $R_{\theta}=1685$. J. Fluid Mech. 420, 47-83.

Beresh, S. J., Clemens, N. T. \& Dolling, D. S. 2002 Relationship between upstream turbulent boundary-layer velocity fluctuations and separation shock unsteadiness. AIAA J. 40, 2412-2422.

Dolling, D. S. \& Murphy, M. T. 1983 Unsteadiness of the separation shock wave structure in a supersonic compression ramp flow field. AIAA J. 21, 1628-1634.

Dolling, D. S. \& OR, C. T. 1985 Unsteadiness of the shock wave structure in attached and separated compression ramp flows. Exp. Fluids 3, 24-32.

Dupont, P., Haddad, C. \& Debieve, J. F. 2006 Space and time organization in a shock-induced separated boundary layer. J. Fluid Mech. 559, 255-277.

Dussauge, J. P., Dupont, P. \& Debieve, J. F. 2006 Unsteadiness in shock wave boundary layer interactions with separation. Aerosp. Sci. Technol. 10, 85-91.

Ganapathisubramani, B., Clemens, N. T. \& Dolling, D. S. 2006 Effects of upstream boundary layer on the unsteadiness of shock induced separation. J. Fluid Mech. 585, 369-394.

Ganapathisubramani, B., Clemens, N. T. \& Dolling, D. S. 2009 Low-frequency dynamics of shock-induced separation in a compression ramp interaction. J. Fluid Mech. 636, 397-425.

HiCKEL, S. 2012 Implicit subgrid-scale modelling for large eddy simulation of compressible flows and shock turbulence interaction Phys. Fluids (submitted).

Hickel, S., Adams, N. A. \& Domaradzki, J. A. 2006 An adaptive local deconvolution method for implicit LES. J. Comput. Phys. 213, 413-436.

Huang, P. G., Coleman, G. N. \& BRAdshaw, P. 1995 Compressible turbulent channel flows: DNS results and modelling. J. Fluid Mech.

Loginov, M., Adams, N. A. \& Zheltovodov, A. 2006 Large-eddy simulation of shockwave/turbulent boundary layer interaction. J. Fluid Mech. 565, 133-169.

Piponniau, S., Dussauge, J. P., Debieve, J. F. \& Dupont, P. 2009 A simple model for low-frequency unsteadiness in shock-induced separation. J. Fluid Mech. 629, 87-108.

Pirozzoli, S. \& GRASSO, F. 2006 Direct numerical simulation of impinging shock wave/turbulent boundary layer interaction at $M a=2.25$. Phys. Fluids 8, 1-17.

Pirozzoli, S., Larsson, J., Nichols, J. W., Bernardini, M., Morgan, B. E. \& LELE, S. K. 2010 Analysis of unsteady effects in shock/boundary layer interaction. In Center for Turbulence Research Proceedings of the Summer Program. Center for Turbulence Research, Stanford University and NASA Ames Research Center, Stanford, CA.

Ringuette, M. J., Martin, M. P. \& Smits, A. J. 2006 Characterization of the turbulence structure in supersonic boundary layers using DNS data. AIAA Paper 2006-3539.

Rowley, C. W., Mezic, I., Bagheri, S., Schlatter, P. \& Henningson, D. S. 2009 Spectral analysis of nonlinear flows. J. Fluid Mech. 641, 1-13.

SCHMID, P. J. 2010 Dynamic mode decomposition of numerical and experimental data. J. Fluid Mech. 656, 5-28. 
SCHMID, P. J. 2011 Applications of the dynamic mode decomposition to experimental data. Exp. Fluids 50, 1123-1130.

SPAlaRT, P. R. 1988 Direct simulation of a turbulent boundary layer up to $R e_{\theta}=1410$. J. Fluid Mech. 187.

Touber, E. \& SANDhAM, N. 2009 Large-eddy simulation of low-frequency unsteadiness in a turbulent shock-induced separation bubble. Theor. Comput. Fluid Dyn. 23, 79-107.

Wu, M. \& MARTin, M. P. 2007 Direct numerical simulation of shockwave and turbulent boundary layer interaction induced by a compression ramp. AIAA J. 45, 879-889.

Zheltovodov, A. A., Trofimov, V. M., Schülein, E. \& Yakovlev, V. N. 1990 An experimental documentation of supersonic turbulent flows in the vicinity of forward- and backward-facing ramps. Tech. Rep. 2030. Inst. Theor. Appl. Mech., USSR Acad. Sci., Novosibirsk. 